\title{
Involvement of the opportunistic pathogen Aspergillus tubingensis in osteomyelitis of the maxillary bone: a case report
}

\author{
Erik Bathoorn ${ }^{1,3^{*}}$, Natalia Escobar Salazar ${ }^{3}$, Shahrzad Sepehrkhouy ${ }^{2}$, Martin Meijer ${ }^{4}$, Hans de Cock ${ }^{3}$ \\ and Pieter-Jan Haas'
}

\begin{abstract}
Background: Aspergillus tubingensis is a black Aspergillus belonging to the Aspergillus section Nigri, which includes species that morphologically resemble Aspergillus niger. Recent developments in species determination have resulted in clinical isolates presumed to be Aspergillus niger being reclassified as Aspergillus tubingensis by sequencing. We present a report of a patient with an osteomyelitis of the maxillary bone with a probable invasive Aspergillus tubingensis infection.

Case presentation: We describe an immune compromised patient suffering from osteomyelitis of the maxillary bone after tooth extraction. The osteomyelitis probably resulted in dentogenic pansinusitis presenting as an acute ethmoiditis. Histologic examination of biopsy samples showed osteomyelitis, and inflammation of the surrounding connective tissue. Cultures of the alveolar wound grew Aspergillus tubingensis. The patient was treated with liposomal amphoterocin B, which was changed to oral treatment with voriconazole based on susceptibility testing (MIC for voriconazole was $1 \mu \mathrm{g} / \mathrm{ml}$ ).

Conclusion: This case shows that Aspergillus tubingensis may have the potential to cause severe invasive infections in immunocompromised hosts. A larger proportion of Aspergillus tubingensis isolates are less susceptible to azoles compared to Aspergillus niger. Therefore, correct species identification and susceptibility testing is crucial for the choice of anti-fungal treatment, screening of azole resistance, and characterization of the pathogenic potential of the various species within Aspergillus section Nigri.
\end{abstract}

Keywords: Aspergillus tubingensis, Aspergillus section nigri, Invasive mould infection, Species identification

\section{Background}

Aspergillus tubingensis is a black Aspergillus species described by Raoul Mosseray in 1934 [1]. This species is found all over the world, grows predominantly on dead plant material, and is food associated [2]. A. tubingensis belongs to the Aspergillus section Nigri, which also includes A. niger, and A. awamori amongst a total of 25 species [2-4]. The species belonging to the Aspergillus section Nigri are phylogenetically closely related. This makes the taxonomy and species determination difficult.

\footnotetext{
* Correspondence: d.bathoorn@umcutrecht.nl

'Department of Medical Microbiology, University Medical Center Utrecht, Postbox 85500, Utrecht, The Netherlands

${ }^{3}$ Institute of Biomembranes, Faculty of Science, Utrecht University, Utrecht, The Netherlands

Full list of author information is available at the end of the article
}

In general, species can be distinguished via a polyphasic approach using morphology, biochemical properties, and molecular data [3,5]; however, most of the species belonging to the Aspergillus section Nigri are morphologically indistinguishable. Several species do have some distinct biochemical properties: nutritional growth conditions and hydrolase differences between the species have been described [6]. Furthermore, production of secondary metabolites is often unique for a species within Aspergillus section Nigri, and could be used for identification [2], but it is not yet possible to differentiate the species solely on metabolic properties.

Due to the above mentioned difficulties in discrimination of species, the most commonly used method for species identification of Aspergillus section Nigri is sequencing.

\section{Biomed Central}


Using calmodulin or $\beta$-tubulin data sequencing data, all Aspergillus section Nigri species can be clearly distinguished [3]. The development of these molecular diagnostic tools has facilitated correct species determination of black Aspergilli.

In this report, we describe a patient with a probable invasive infection with $A$. tubingensis, and discuss the clinical importance of A. tubingensis and its correct species determination.

\section{Case presentation}

A 19-year-old male patient born in the Netherlands (Moroccan parents) presented with fever, rhinitis and progressive pain behind the left eye. His recent medical record included a second non-myeloablative stem cell transplantation for graft failure resulting in relapse of paroxysmal nocturnal haemoglobinuria 3 weeks prior to presentation. He had undergone a maxillary tooth extraction 47 days prior to presentation. The alveolar wound did not heal due to osteomyelitis, for which he had a biopsy of the maxillary bone 23 days prior to presentation. Histological examination of the biopsy samples showed necrotic bone tissue. The surrounding connective tissue was infiltrated by leukocytes. Yeast cells, and focal bacterial colonies were noted. He was treated empirically with amoxicillin/clavulanic acid orally, which had been started 3 days prior to presentation, and the immunosuppressive drugs cyclosporine, mycophenolic acid, and prednisone. With this treatment, the patient was clinically deteriorating.

Physical examination revealed a body temperature of $37.9^{\circ} \mathrm{C}$ and normal vital signs. A purulent wound in the mouth resulting from the biopsy, left pre-orbital swelling, and painful cervical lymphadenopathy were observed. Laboratory tests showed increased C-reactive protein at $346 \mathrm{mg} / \mathrm{L}$, leucopenia (leukocytes $<0.1 \times 10^{9} / \mathrm{L}$ ), thrombopenia (thrombocytes $32 \times 10^{9} / \mathrm{L}$ ) and normocytic anaemia (haemoglobin $5.51 \times 10^{9} / \mathrm{L}$ ). Computed tomography of the head showed pre-orbital swelling with induration of the subcutis, swollen mucosa of the sphenoid, and maxillary sinus, and total opacification of the left ethmoid sinus (Figure 1). Since a direct surgical approach was impossible due to extensive bleeding of the swollen mucosa, an antrostomy was performed and sinus secretions were thoroughly aspirated. Cultures of purulent fluid from the biopsy wound in the mouth on Sabouraud dextrose agar at $37.0^{\circ} \mathrm{C}$ grew a black Aspergillus, morphologically resembling $A$. niger. Cultures of the aspirated secretion from sinus cavities were negative for bacteria and fungi. Results of the histological examination of mucosal lining samples of the nasal sinus were consistent with chronic sinusitis. The result of molecular identification of the black Aspergillus by sequencing of the $\beta$-tubilin gene was $A$. tubingensis Mosseray (CBS-KNAW, Fungal Biodiversity Centre,

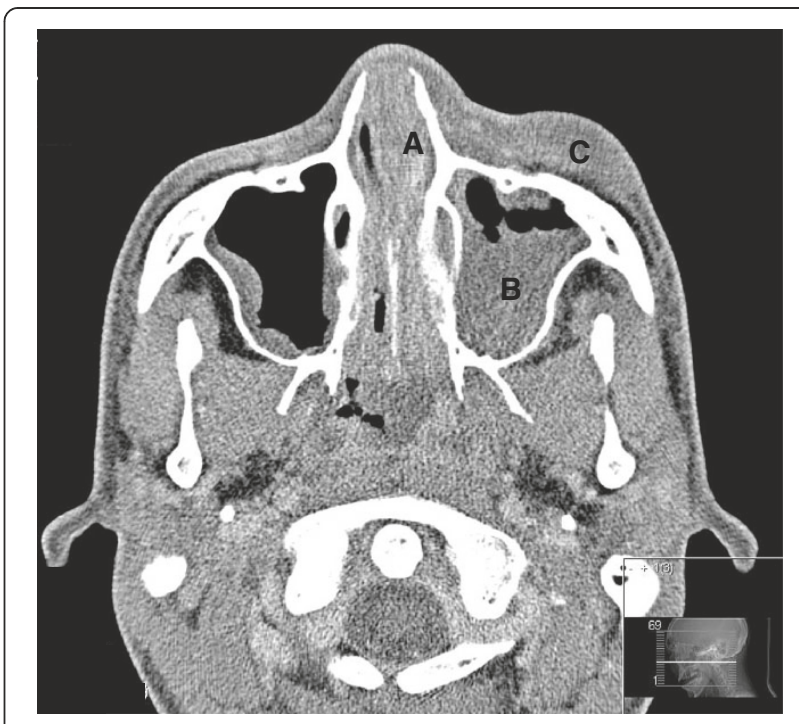

Figure 1 Computed tomography of the head showing A: total opacification of the ethmoid sinus; B: swollen mucosa of the maxillary sinus; C: pre-orbital swelling.

Utrecht, The Netherlands, strain CBS 133792; GenBank accession number KC163802). Figure 2 shows the phylogenetic relationships among Aspergillus section Nigri species, including our isolated strain (Table 1). Antifungal susceptibility testing by microdilution showed that the strain had MIC values to voriconazole of $1 \mu \mathrm{g} / \mathrm{mL}$, to posaconazole of $0.25 \mu \mathrm{g} / \mathrm{mL}$, to itraconazole of $0.25 \mu \mathrm{g} / \mathrm{mL}$, to fluconazole of $>24 \mu \mathrm{g} / \mathrm{mL}$, to anidulafungin of 0.125 $\mu \mathrm{g} / \mathrm{mL}$, to amphothericin B of $0.5 \mu \mathrm{g} / \mathrm{mL}$, and to flucytosine of $2 \mu \mathrm{g} / \mathrm{mL}$ [7].

The patient was treated with liposomal amphothericin B $5 \mathrm{mg} / \mathrm{kg}$ for 6 weeks and imipinem $500 \mathrm{mg} /$ qid for 2 weeks intravenously, followed by prolonged treatment with voriconazole $300 \mathrm{mg} /$ tid oral for 4 months. With this treatment the patient recovered from the sinusitis, but passed away later on that year due to BK virus encephalitis.

In summary, we describe an immunocompromised patient suffering from osteomyelitis of the maxillary bone with a probable invasive $A$. tubingensis infection of the surrounding connective tissue after tooth extraction. This probably resulted in dentogenic pansinusitis presenting as an acute ethmoiditis.

\section{Conclusions}

Species belonging to the Aspergillus section Nigri have been identified as opportunistic pathogens, particularly in cases of otitis and sinusitis. Of the 25 species in section Nigri, A. niger has most often been described as the cause of infection, however, in most cases species determination was based only on morphology [8]. Osteomyelitis caused by Aspergillus section Nigri is very rare [9], 


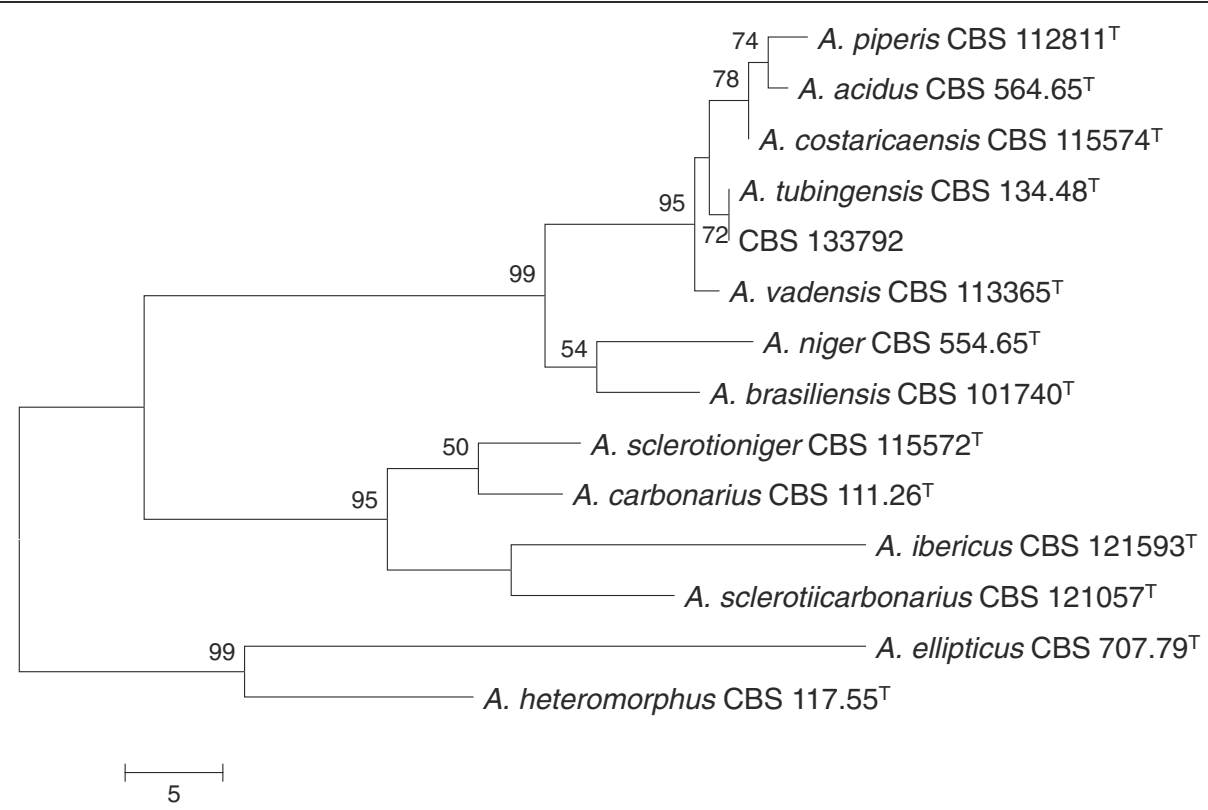

Figure 2 Maximum Parsimony tree based on $\beta$-tubulin sequence data of type strains of Aspergillus section Nigri, including the Aspergillus tubingensis (CBS 133792) strain isolated from the presented patient. Numbers above branches represent bootstrap values.

and by $A$. tubingensis has to the best of our knowledge not been described. We isolated $A$. tubingensis from a purulent wound localized on the surface of the infected maxillary bone. The immunocompromised patient deteriorated while he was treated with antibiotics, and improved after antimycotics were added. Therefore we consider this case as a probable invasive aspergillosis. Alternatively, the infection may have been caused by yeasts, which were noted in the necrotic tissue sampled

Table 1 List of Genbank numbers

\begin{tabular}{ll}
\hline Name: & GenBank number: \\
\hline A. acidus CBS 564.65 T & FJ629280 \\
A. brasiliensis CBS 101740 T & FJ629272 \\
A. carbonarius CBS 111.26 T & FJ629276 \\
A. costaricaensis CBS 115574 T & FJ629277 \\
A. ellipticus CBS 707.79 T & FJ629279 \\
A. heteromorphus CBS 117.55 T & FJ629284 \\
A. ibericus CBS 121593 T & AM419748 \\
A. niger CBS 554.65 T & FJ629288 \\
A. piperis CBS 112811 T & FJ629303 \\
A. sclerotiicarbonarius CBS 121057 T & EU159229 \\
A. sclerotioniger CBS 115572 T & FJ629304 \\
A. tubingensis CBS 134.48 T & FJ629305 \\
CBS 133792 & KC163802 \\
A. vadensis CBS 113365 T & FJ629319
\end{tabular}

List of GenBank numbers of isolates from Aspergillus section Nigri that were used for the Maximum Parsimony tree, including the Aspergillus tubingensis (CBS 133792) strain isolated from the presented patient. during the debridement 23 days prior to presentation. We can not rule out involvement of yeasts as well, however, the cultures of the purulent fluid samples taken from the bone biopsy wound at presentation were negative for yeasts.

The subsequent pansinusitis of the patient and acute ethmoiditis were probably dentogenic, since the purulent mouth wound after biopsy was ipsilateral to the preorbital swelling. Problematic wound healing of the alveolus after tooth extraction often causes sinusitis due to fistula of the maxillary sinus and contamination with dental microbial flora [10]. Alternatively, A. tubingensis could have invaded the sinus and directly cause the sinusitis. However, cultures of aspirated secretion from the sinus cavities were negative, and no fungal strains were found with histological examination of biopsies from the mucosa of the sinus cavities.

In recent studies, clinical isolates presumed as $A$. niger species have been re-classified by sequencing as $A$. awamori and A. tubingensis isolates [11-14]. Sequencing of Aspergillus isolates from transplant patients with invasive aspergillosis showed that in 9\% of cases the causative agent belonged to Aspergillus section Nigri, and 32\% of these isolates were determined as A. tubingensis [15]. These studies show the potential of $A$. tubingensis to cause invasive infections.

Differentiating between $A$. tubingensis and A. niger also allows for screening for azole resistance. Thus far, studies on susceptibility patterns suggest that these may not be the same for both the species. About $40-50 \%$ of Aspergillus section Nigri strains have a MIC $>1.0 \mu \mathrm{g} / \mathrm{ml}$ 
to voriconazol, and $70-80 \%$ to itraconazol. A. tubingensis strains more often have higher MICs to itraconazole and voriconazole $[16,17]$. Since there are no clinical breakpoints for Aspergillus section Nigri available, it is uncertain how we should interpret these MIC values. Recently, clinical breakpoints have been presented for A. fumigatus, proposing MICs $\leq 0.5 \mu \mathrm{g} / \mathrm{ml}$ for voriconazole and itraconazole as susceptible [18]. Therefore, in our hospital we start empirical treatment with liposomal amphothericin B in case of infection with a black Aspergillus, and change to azole therapy based on the results of species determination by sequencing and antifungal susceptibility testing.

In conclusion, the presented case shows that A. tubingensis may cause severe invasive infections in immunocompromised hosts. Recent developments in species determination within the Aspergillus section Nigri have resulted in a trend in recognizing $A$. tubingensis as an important opportunistic pathogen. Species determination of clinical Aspergillus section Nigri isolates by sequencing and antifungal susceptibility testing are both crucial for determining appropriate antifungal therapy, epidemiological data on susceptibility patterns, and the pathogenic potential of the various species.

\section{Consent}

Written informed consent was obtained from the patient for publication of this Case report and any accompanying images. A copy of the written consent is available for review by the Series Editor of this journal.

\section{Competing interests}

The authors declare that they have no competing interests.

\section{Author's contributions}

EB collected the clinical data and drafted of the manuscript. NS reviewed the diagnostic tools for black Aspergillus species and helped with the draft of the manuscript. SS carried out the histological examinations. MM performed the sequencing and provided the Maximum Parsimony tree. $\mathrm{HC}$ provided the sequence, contributed to the concept, and edited the manuscript. PH supervised the clinical case interpretation, participated in the coordination and concept of the manuscript, and helped with the draft of the manuscript. All authors read and approved the manuscript.

\section{Author details}

${ }^{1}$ Department of Medical Microbiology, University Medical Center Utrecht, Postbox 85500, Utrecht, The Netherlands. 'Department of Pathology, University Medical Center Utrecht, Utrecht, The Netherlands. ${ }^{3}$ Institute of Biomembranes, Faculty of Science, Utrecht University, Utrecht, The Netherlands. ${ }^{4}$ Applied and Industrial Mycology/Identification Service, CBSKNAW Fungal Biodiversity Centre, Utrecht, the Netherlands.

Received: 16 April 2012 Accepted: 28 January 2013

Published: 1 February 2013

\section{References}

1. Mosseray R: Les aspergillus de la section "Niger" Thom et Church. La Cellule Journal 1934, 43:203-285.

2. Perrone G, Varga J, Susca A, Frisvad JC, Stea G, Kocsube S, et al: Aspergillus uvarum sp. nov., an uniseriate black Aspergillus species isolated from grapes in Europe. Int J Syst Evol Microbiol 2008, 58:1032-1039.
3. Samson RA, Noonim P, Meijer M, Houbraken J, Frisvad JC, Varga J: Diagnostic tools to identify black aspergilli. Stud Mycol 2007, 59:129-145.

4. Perrone G, Stea G, Epifani F, Varga J, Frisvad JC, Samson RA: Aspergillus niger contains the cryptic phylogenetic species A. awamori. Fungal Biol 2011, 115:1138-1150.

5. Samson RA, Varga J: What is a species in Aspergillus? Med Mycol 2009, 47(Suppl 1):S13-S20.

6. Meijer M, Houbraken JA, Dalhuijsen S, Samson RA, de Vries RP: Growth and hydrolase profiles can be used as characteristics to distinguish Aspergillus niger and other black aspergilli. Stud Mycol 2011, 69:19-30

7. Subcommittee on Antifungal Susceptibility Testing of the ESCMID European Committee for Antimicrobial Susceptibility Testing: EUCAST Technical Note on the method for the determination of broth dilution minimum inhibitory concentrations of antifungal agents for conidia-forming moulds. Clin Microbiol Infect 2008, 14:982-984.

8. Munguia R, Daniel SJ: Ototopical antifungals and otomycosis: a review. Int J Pediatr Otorhinolaryngol 2008, 72:453-459.

9. Winslow CP, Dichard A, McGuire KA: Osteomyelitis of the temporomandibular joint. Am J Otolaryngol 2001, 22:142-145.

10. Abrahams JJ, Berger SB: Oral-maxillary sinus fistula (oroantral fistula): clinical features and findings on multiplanar CT. AJR Am J Roentgenol 1995, 165:1273-1276.

11. Szigeti G, Sedaghati E, Mahmoudabadi AZ, Naseri A, Kocsube S, Vagvolgyi C, et al: Species assignment and antifungal susceptibilities of black aspergilli recovered from otomycosis cases in Iran. Mycoses 2012, 55:333-338.

12. Arabatzis M, Kambouris M, Kyprianou M, Chrysaki A, Foustoukou M, Kanellopoulou $M$, et al: Polyphasic identification and susceptibility to seven antifungals of 102 Aspergillus isolates recovered from immunocompromised hosts in Greece. Antimicrob Agents Chemother 2011, 55:3025-3030

13. Szigeti G, Kocsube S, Doczi I, Bereczki L, Vagvolgyi C, Varga J: Molecular identification and antifungal susceptibilities of black Aspergillus isolates from otomycosis cases in Hungary. Mycopathologia 2012, 174(2):143-147.

14. Hendrickx M, Beguin H, Detandt M: Genetic re-identification and antifungal susceptibility testing of Aspergillus section Nigri strains of the BCCM/IHEM collection. Mycoses 2012, 55:148-155.

15. Balajee SA, Kano R, Baddley JW, Moser SA, Marr KA, Alexander BD, et al: Molecular identification of Aspergillus species collected for the transplant-associated infection surveillance network. J Clin Microbio/ 2009, 47:3138-3141.

16. Alcazar-Fuoli L, Mellado E, Alastruey-Izquierdo A, Cuenca-Estrella M, Rodriguez-Tudela JL: Species identification and antifungal susceptibility patterns of species belonging to Aspergillus section Nigri. Antimicrob Agents Chemother 2009, 53:4514-4517.

17. Howard SJ, Harrison E, Bowyer P, Varga J, Denning DW: Cryptic species and azole resistance in the Aspergillus niger complex. Antimicrob Agents Chemother 2011, 55:4802-4809.

18. Meletiadis J, Mavridou E, Melchers WJ, Mouton JW, Verweij PE: Epidemiological cutoff values for azoles and Aspergillus fumigatus based on a novel mathematical approach incorporating cyp51A sequence analysis. Antimicrob Agents Chemother 2012, 56(5):2524-2529.

doi:10.1186/1471-2334-13-59

Cite this article as: Bathoorn et al:: Involvement of the opportunistic pathogen Aspergillus tubingensis in osteomyelitis of the maxillary bone: a case report. BMC Infectious Diseases 2013 13:59. 